\title{
Effect of the solar wind conditions on the ionospheric equivalent current systems
}

\author{
J. J. Zhang ${ }^{1,2}$, C. Wang ${ }^{1}$, B. B. Tang ${ }^{1}$, and H. $\mathbf{L i}^{1}$ \\ ${ }^{1}$ State Key Laboratory of Space Weather, Center for Space Science and Applied Research, Chinese Academy of Sciences, \\ Beijing 100190, China \\ ${ }^{2}$ University of the Chinese Academy of Sciences, Beijing 100049, China
}

Correspondence to: J. J. Zhang (jjzhang@spaceweather.ac.cn)

Received: 16 November 2012 - Revised: 18 January 2013 - Accepted: 1 March 2013 - Published: 14 March 2013

\begin{abstract}
We employ a global magnetohydrodynamics (MHD) model, namely the PPMLR-MHD model, to investigate the effect of the solar wind conditions, such as the interplanetary magnetic field (IMF) clock angle, southward IMF magnitude and solar wind speed, on the average pattern of the ionospheric equivalent current systems (ECS). A new method to derive ECS from the MHD model is proposed and applied, which takes account of the oblique magnetic field line effects. The model results indicate that when the IMF is due northward, the ECS are very weak while the current over polar region is stronger than the lower latitude; when the IMF rotates southward, the two-cell current system dominates, the eastward electrojet on the afternoon sector and the westward electrojet on the dawn sector increase rapidly while the westward electrojet is stronger than the eastward electrojet. Under southward IMF, the intensity of the westward electrojet and eastward electrojet both increase with the increase of the southward IMF magnitude and solar wind speed, and the increase is very sharp for the westward electrojet. Furthermore, we compare the geomagnetic perturbations on the ground represented by the simulated average ECS with the observation-based statistical results under similar solar wind conditions. It is found that the model results generally match with the observations, but the underestimation of the eastward equivalent current on the dusk sector is the main limitation of the present model.
\end{abstract}

Keywords. Ionosphere (Electric fields and currents; Modeling and forecasting) - Magnetospheric physics (Solar windmagnetosphere interactions)

\section{Introduction}

Ionospheric equivalent current systems (ECS) are a convenient way to represent detailed features of global magnetic activity including intensity, location and pattern. They overcome the limitation of many geomagnetic indices, such as $\mathrm{Kp}$, ap, AE and Dst, which are intended to describe only the intensity information of a particular geomagnetic latitudinal band (Kamide et al., 1976). Under many circumstances, ECS are a good approximation of the ionospheric currents shedding light on the spatial and temporal varying behavior of the basic processes in the ionosphere although they do not equal the true horizontal ionospheric current systems (Untiedt and Baumjohann, 1993).

From the viewpoint of space weather, the ECS are of great interest because they relate to the geomagnetic induction phenomena which could cause problems on technological facilities on the ground. Driven by rapid variation of the nearspace current systems, the geoelectric field induces on the surface of the earth producing geomagnetically induced currents (GIC) in the man-made long conductor systems. When GICs are large enough to exceed the thresholds of the systems, severe electric blackout, communication outage, and corrosion of oil and gas pipelines would happen (Boteler and Pirjola, 1998), leading to huge economic and social losses. Attempts have been made to model the geomagnetic induction effects from the ECS with the ultimate aim of mitigating or avoiding the harmful effects caused by GICs. Pulkkinen et al. (2003) calculated geoelectric fields at some Baltic Electromagnetic Array Research (BEAR) sites from ECS determined by the method of spherical elementary current system by using the complex image method (Pirjola and Viljanen, 
1998). At some sites the electric fields they calculated are in good agreement with the measurement. From the ECS derived in a global magnetohydrodynamics (MHD) model, Zhang et al. (2012) have simulated GIC flowing through a transformer of the Pirttikoski (PIR) substation in the Finnish high-voltage power system during a space weather event of 1999. They compared the modeled GIC with the GIC recording and found that the simulation reproduced the main features of the GIC signals for the event they investigated.

Global MHD simulations develop rapidly for several recent decades. Many three-dimensional (3-D) MHD codes have been developed to simulate the global behavior of the solar wind-magnetosphere-ionosphere coupling system in literature, for example, the Lyon-Fedder-Mobarry (LFM) code (Lyon et al., 1998), the BATS-R-US code of the Michigan group (Powell et al., 1999), the European GUMICS code (Janhunen, 1996), the PPMLR-MHD simulation code (Hu et al., 2007) and so on. Such models are essential tools to understand the geospace environment and the plasma physical processes in it, since in situ measurements are often too sparse to enable a unique interpretation of the data, and the complexity of the space environment limits our theoretical understanding as well (Raeder et al., 2001b). In addition, global MHD models are crucial for space weather applications, especially under extreme solar wind and interplanetary magnetic field (IMF) conditions. When the empirical models are unable to make predictions under the extreme conditions which are often outside the range of validity, MHD models can still make physical principle-based predictions.

Deriving ECS from a global MHD simulation model is of significant in both magnetosphere-ionosphere coupling and space weather study. Wang et al. (2011) have successfully reproduced the ECS during two successive isolated substorms by using the global PPMLR-MHD model (Hu et al., 2007). Driven by the upstream solar wind, however, the general average ECS pattern as a function of the solar wind and IMF has not been investigated so far. With respect to space weather, the average ECS pattern could give us a hint about where the GIC hazards would be most likely to occur under specific solar wind and IMF conditions, thus a rough but much advance prediction of the vulnerable area of GIC hazards could be made, since large $d B / d t$ values occur predominantly during westward ionospheric electrojets (Viljanen and Tanskanen, 2011). On the other hand, average patterns of the ionospheric electric potentials and field aligned currents (FAC) as a function of solar wind conditions have been studied for many years and their patterns for any arbitrary solar wind conditions can be reproduced easily nowadays (e.g., Ridley et al., 2000; Papitashvili et al., 2002; Weimer, 2005b), but so far there have been few publications dealing with the calculation of global-scale horizontal ionospheric currents as a function of the solar wind and IMF (Friis-Christensen et al., 1985; Weimer, 2005a). As a good approximation of the horizontal ionospheric currents, derivation of the average ECS as a function of the solar wind conditions will have an impor- tant role in developing an understanding of the physical processes occurring in the coupled solar wind-magnetosphereionosphere system.

In this study we will derive the average ECS from the global PPMLR-MHD model and investigate how the IMF clock angle, southward IMF magnitude and solar wind speed influence the pattern and intensity of the ECS. Furthermore, we would also like to find a way to verify the model results. The ECS are usually the representation of the geomagnetic perturbation on earth's surface, so comparisons of the ECS derived from the simulation and the observation are equivalent to comparing the geomagnetic perturbations on the ground calculated from the simulated ECS with the observation. Weimer et al. (2010) mapped the statistical geomagnetic perturbations as a function of the IMF and solar wind conditions by using a spherical harmonics fits technique. The geomagnetic data they used was collected from 104 geomagnetic observatories in the Northern Hemisphere at geomagnetic latitude above $40^{\circ}$ during years 1998 to 2001 . We will calculated the three components of geomagnetic variations on the ground from the simulated average ECS and then compare them with the statistical results from Weimer et al. (2010) under similar solar wind and IMF conditions.

In addition, an approach to deriving the ECS from the global MHD model, which includes the oblique magnetic field line effects is proposed and applied in this study. In order to get ECS from a global MHD model for the high latitude region, previous work usually split the ionospheric currents into divergency-free toroidal current and curl-free poloidal current, and adopted the toroidal current as the equivalent current in a global MHD model under the assumption of radial geomagnetic field lines (Raeder et al., 2001a; Wang et al., 2011). Under this assumption the geomagnetic perturbations produced by ionospheric poloidal current and field-aligned current (FAC) cancel each other (Fukushima, 1976; Kamide et al., 1981). In reality, the field lines are not radial at high latitude regions, the incline of the field lines should be considered for more accurate estimations. In this study we propose and apply an approach to deriving the ECS in the global MHD model, which takes the effects of the ionospheric poloidal current and the FAC into account.

In the remainder of this paper we will first introduce the numerical model and the approach to derive the ECS; we will then present the simulated average ECS under various solar wind and IMF conditions and validation of the results; discussion and summary will be given last.

\section{Methodology}

\subsection{The numerical model}

The numerical model used in this study is the global PPMLR-MHD model (Hu et al., 2007), which is the same as our previous work (Wang et al., 2011), here we give 
only a brief description. The PPMLR-MHD code solves ideal MHD equations for the solar wind-magnetosphereionosphere coupling system. The numerical box is taken to be $-300 R_{\mathrm{E}} \leq x \leq 30 R_{\mathrm{E}},-150 R_{\mathrm{E}} \leq y, z \leq 150 R_{\mathrm{E}}$ with the smallest grid spacing of $0.4 R_{\mathrm{E}}$ in GSM coordinates in the study. To avoid the complexity associated with the plasmasphere and strong magnetic field, an inner magnetosphere boundary is set at $r=3 R_{\mathrm{E}}$, and an electrostatic ionosphere is set at $r=1.017 R_{\mathrm{E}}$, a magnetosphere-ionosphere electrostatic coupling model is imbedded between the inner boundary and the ionosphere. To calculate the conductance tensor of the ionosphere, two models are applied together. For the contribution from the solar EUV radiation, we use a model in which the conductance depends on the solar flux $F_{10.7}$ and solar zenith angle $\chi$ (Moen and Brekke, 1993). For the auroral region, the model developed by Ahn et al. (1998) is used, in which the conductances are empirically derived from the geomagnetic disturbances. The geomagnetic perturbation produced from the model is the input of the conductance model in the simulation, while an arbitrary weak ionospheric conductance is given at the very beginning of the simulation. Since the state of the system we are interested in has been obtained after a long time, the choice of the arbitrary conductance does not influence the result significantly. The Hall and Pedersen conductance over the dark polar cap region and subauroral region are set to constants $2.0 \mathrm{~S}$ and $1.0 \mathrm{~S}$, respectively, regardless of the magnetic perturbation level.

\subsection{The method to derive ECS}

In order to include the influence of the ionospheric poloidal current and the FAC on the ECS, we first define fictitious magnetometer sites at the ground grid points which are every $1^{\circ}$ in magnetic latitude and $1 \mathrm{~h}$ in magnetic local time. Then we calculate magnetic variations at each site produced by the ionospheric toroidal current $J_{\mathrm{T}}$, the poloidal current $J_{\mathrm{P}}$ at high latitude region above $60^{\circ}$ magnetic latitude and the data gap region FAC $J_{\|}$by using the Biot-Savart's law:

$\delta \boldsymbol{B}(\boldsymbol{R})=\frac{\mu_{0}}{4 \pi} \int_{\mathrm{S}} \boldsymbol{J}\left(\boldsymbol{r}^{\prime}\right) \times \frac{\boldsymbol{R}-\boldsymbol{r}^{\prime}}{\left|\boldsymbol{R}-\boldsymbol{r}^{\prime}\right|^{3}} d S$

$\delta \boldsymbol{B}(\boldsymbol{R})=\frac{\mu_{0}}{4 \pi} \int_{V} \boldsymbol{J}\left(\boldsymbol{r}^{\prime}\right) \times \frac{\boldsymbol{R}-\boldsymbol{r}^{\prime}}{\left|\boldsymbol{R}-\boldsymbol{r}^{\prime}\right|^{3}} d V$,

where $\boldsymbol{J}\left(\boldsymbol{r}^{\prime}\right)$ is the current source, $\boldsymbol{r}^{\prime}$ denotes the position of the current source, $\boldsymbol{R}$ is the location of the point of interest on the ground, $\mu_{0}$ is the magnetic permeability of free space. Equation (1) is used for the geomagnetic disturbance produced by the horizontal ionospheric current $J_{\mathrm{T}}$ and $J_{\mathrm{P}}$. Equation (2) is used for the geomagnetic disturbance produced by $J_{\|}$, the calculation takes account of the tilted FAC from ionosphere until $3 R_{\mathrm{E}}$ (inner magnetosphere boundary of the model).
The data gap region is the region between the inner boundary and the ionosphere mentioned in Sect. 2.1, in which $J_{\|}$ is mapped from the inner magnetosphere boundary along earth's dipole magnetic field lines. To get the information of the current in this region, we utilize the relation:

$J_{\|} / B_{\mathrm{d}}=$ constant

to calculate $J_{\|}$at any point of the region along the field lines from the current at the inner boundary, where $J_{\|}$and $B_{\mathrm{d}}$ represent the FAC and magnetic field magnitude at any position of the gap region along a field line, respectively.

Lastly, the geomagnetic perturbations at the fictitious magnetometer sites are input to a geomagnetic inversion algorithm to derive the ECS in the ionosphere at radius $r=$ $1.017 R_{\mathrm{E}}$. The algorithm is based on the principles as follows. In the lower atmosphere, where electric current flows are negligible, the magnetic variation can be expressed in terms of a magnetic potential $\Phi$ as

$\boldsymbol{B}=-\operatorname{grad} \Phi$,

and the equivalent current is related to the external portion of $\Phi$ by a straightforward mathematical relation (Chapman and Bartels, 1940):

$J_{n}(r, \theta, \phi)=-\frac{1}{\mu_{0}} \frac{2 n+1}{n+1}\left(\frac{r}{r_{0}}\right)^{n} \Phi_{n}^{\mathrm{e}}\left(r_{0}, \theta, \phi\right)$,

where $\Phi_{n}^{\mathrm{e}}$ represents terms in the spherical harmonic series for the external portion of the magnetic potential at radius $r_{0}$ at the surface of earth, and $J_{n}$ represents the corresponding term in the series for the equivalent current function in the ionosphere at radius $r$. The magnetic potential is determined from the calculated geomagnetic perturbations at the fictitious magnetometer sites, then we utilize the external portion of $\Phi$ and Eq. (5) to obtain the ECS.

This method about derivation of the ECS from the geomagnetic perturbations was also adopted in the famous KRM algorithm (see Kamide et al., 1981, for details).

\subsection{The input IMF and solar wind parameters}

The global MHD model is driven by the solar wind and IMF at the inflow boundary. The input parameters include the three components of the IMF, $B_{\mathrm{x}}, B_{\mathrm{y}}, B_{\mathrm{z}}$, three components of solar wind velocity, $V_{\mathrm{x}}, V_{\mathrm{y}}, V_{\mathrm{z}}$, solar wind plasma density, $N$ and plasma temperature, $T$. In order to get average ECS under certain interplanetary conditions, the MHD parameters at the inflow boundary are changed to certain solar wind and IMF parameters after the system reaches the quasi-steady state, and the parameters remain constant in each simulation run for about $3 \mathrm{~h}$ (physical time). Then we take the averaged ECS over the period of calculation as the ECS under certain IMF and solar wind conditions. 


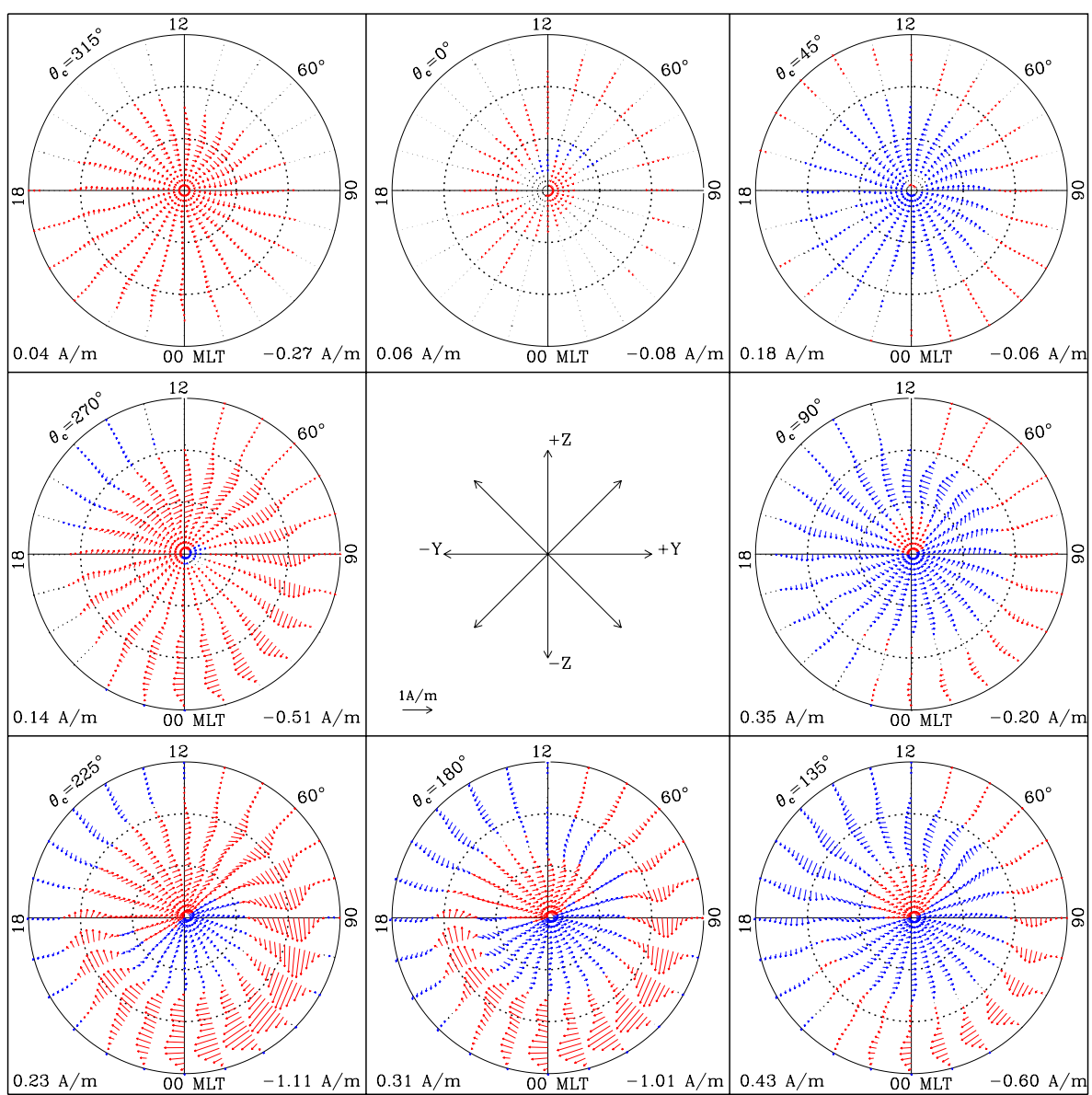

Fig. 1. Average ECS at 8 different IMF clock angles with increments of $45^{\circ}$. The scale length of current vector is shown at the bottom of the center box. Latitude circles are plotted at $10^{\circ}$ intervals, the outer boundary of each plot is at $60^{\circ}$ latitude.

\section{Results}

We examine in this section how the average ECS over the Northern Hemisphere vary with the change of the IMF clock angle, the southward IMF magnitude and the solar wind speed. Then we verify the simulation results by comparing geomagnetic perturbations.

\subsection{ECS as a function of IMF clock angle}

The zero clock angle is defined in the direction of the positive $Z$ with $B_{\mathrm{y}}=0$, the angle $\theta_{\mathrm{c}}$ increases as the IMF rotates clockwise in the GSM Y-Z plane. In order to investigate the influence of the IMF clock angle, the other IMF and solar wind parameters keep constant in all of the runs: the transverse IMF magnitude $B_{\mathrm{T}}\left(B_{\mathrm{T}}=\sqrt{B_{\mathrm{y}}^{2}+B_{\mathrm{z}}^{2}}\right)$ is set to $10 \mathrm{nT}$; solar wind velocity $V_{\mathrm{x}}$ is $450 \mathrm{~km} \mathrm{~s}^{-1}$; solar wind number density $N$ is $7.5 \mathrm{~cm}^{-3}$; plasma temperature $T$ is a typical value of $10^{5} \mathrm{~K} ; B_{\mathrm{x}}, V_{\mathrm{y}}$, and $V_{\mathrm{z}}$ are 0 .

\subsubsection{Simulation results}

We show the average ECS patterns at 8 different IMF clock angles in Fig. 1. The peak values of the eastward current and westward current are given in the bottom corners in each polar plot, with the eastward current as positive. For each single pattern, the equivalent current vectors are plotted at grid points every $1^{\circ}$ in magnetic latitude and $1 \mathrm{~h}$ in magnetic local time. The red vectors denote the westward current, and the blue ones represent the eastward current. The length of the vector represents the current intensity, the same scale length of vector is adopted for all patterns.

Figure 1 shows that the current is very weak when the IMF is pointing northward, but the current over the polar cap region is somewhat larger than that over the lower latitude region. As the clock angle rotates to $90^{\circ}$ clockwise, the current increases slightly, the eastward current distributes over most of the polar region, and the westward current mainly distributes on the dawn sector between $60^{\circ}$ to $70^{\circ}$ latitude with a lower intensity. When the IMF rotates southward, the eastward electrojet at the afternoon sector and the westward 


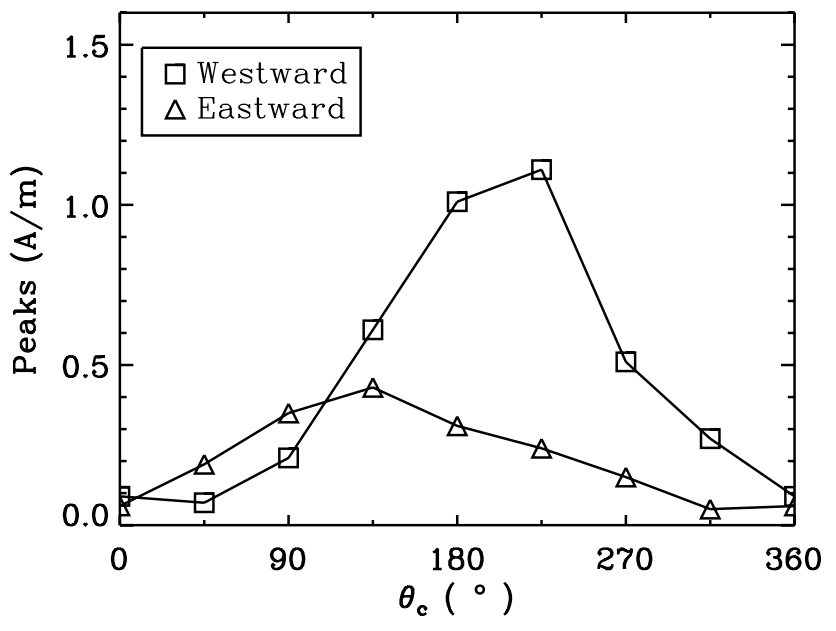

Fig. 2. Peak values of the westward currents and eastward currents as a function of the IMF clock angle $\theta_{\mathrm{c}}$. Square and triangle symbols represent westward and eastward peak values, respectively.

electrojet on the dawn sector develop evidently and they mainly distribute between $60^{\circ}$ to $75^{\circ}$ latitude, the westward current is stronger than that of the eastward. As the IMF clock angle rotates from $225^{\circ}$ to $315^{\circ}$ clockwise, the intensities of both the eastward and westward currents decrease. The influence of IMF $B_{\mathrm{y}}$ on the ECS patterns can be clearly seen from the comparison of the patterns at $\theta_{\mathrm{c}}=90^{\circ}(+\mathrm{y}-$ direction) and at $\theta_{\mathrm{c}}=270^{\circ}$ (-y-direction). The two patterns are generally mirror images with a reverse sign. The dawndusk asymmetry is noticeable for each pattern, which may be mainly the results of the asymmetry between the dusk cell and dawn cell of the electric potential pattern. By checking the electric potential patterns in simulation under these IMF conditions, we find that in the Northern Hemisphere, when the IMF points in the $+y$-direction, the negative dusk cell is more large and round, while the positive dawn cell is more crescent shaped with a smaller absolute magnitude of the potential peak; when the IMF points into the $-y$-direction the situation is reversed. That is consistent with what Pettigrew et al. (2010) described which is based on five years of SuperDARN data. In addition, comparison of patterns at $\theta_{\mathrm{c}}=135^{\circ}$ $\left(B_{\mathrm{y}}>0\right)$ and at $\theta_{\mathrm{c}}=225^{\circ}\left(B_{\mathrm{y}}<0\right)$ shows that a negative IMF $B_{\mathrm{y}}$ condition seems to favor a nightside westward electrojet when the IMF is southward.

The peak values of the westward and eastward currents are extracted and Fig. 2 shows how they evolve as the IMF clock angle rotates. We can see that the two kinds of peak values increase as the IMF rotates southward, and after they reach their maximum, respectively, they start to decline as the IMF rotates clockwise continuously. The westward peak values are generally larger than the eastward peak values.

\subsubsection{Comparison with the statistical maps of geomag- netic perturbations}

To verify the simulation results, we calculate the three components of the geomagnetic perturbations on the surface of earth by using Biot-Savart's law with the simulated ECS presented in Fig. 1. Under similar IMF and solar wind conditions, we make comparison between our simulation results and the observation-based statistical results of Weimer et al. (2010). Figures 3, 4 and 5 show the patterns of the three geomagnetic components (northward, eastward and vertical) as a function of the IMF clock angle $\theta_{\mathrm{c}}$. They correspond to Figs. 9, 10 and 11 in the work of Weimer et al. (2010), respectively. The graph format is generally the same for these two works, but it should be noticed that the outer boundary of each contour map in our simulation work is $60^{\circ}$ magnetic latitude since the main features of geomagnetic filed perturbations distribute above this latitude, while the outer boundary is $40^{\circ}$ magnetic latitude in the statistical work. The intensities of the perturbations are characterized by the color interpreted in the color bar, the northward, eastward and downward are specified as positive. The solar wind and IMF conditions are similar in the two works. The transverse IMF magnitude $\left(B_{\mathrm{T}}=10 \mathrm{nT}\right)$, solar wind speed $\left(V_{\mathrm{x}}=450 \mathrm{~km} \mathrm{~s}^{-1}\right)$ are the same in the two works and the solar wind density number $\left(N=7.5 \mathrm{~cm}^{-3}\right)$ in the simulation approximates to the average value of the solar wind plasma density of the database used in the statistical work. The dipole tilt angle in the simulation is $0^{\circ}$ and corresponds to yearly average tilt angle or equinox, while in Figs. 9-11 of Weimer et al. (2010) the observations are around the summer solstice. Though the intensity of the geomagnetic perturbations change with season, Fig. 14 in Weimer et al. (2010) implies that the perturbations evolve in a similar way as a function of the IMF clock angle in different season. So qualitative comparison will be reasonable.

The regions of the northward and southward perturbations just correspond to that of the eastward and westward equivalent currents, respectively. For the northward component (Fig. 3), when IMF is northward the geomagnetic perturbations are weak and distribute over the polar region; when the IMF rotates to southward, the southward perturbation on the dawn side and the northward perturbation on the dusk side develop greatly, the regions of most intense perturbations are located between $60^{\circ}$ and $70^{\circ}$ latitude. The peak values of the southward perturbations are located at the sector before dawn while that of the northward perturbations are located in afternoon sector. The distribution of the northward and southward magnetic field perturbations and the locations of their peak values at each IMF clock angle generally match with the statistical results. The eastward component (Fig. 4) of the magnetic field variations is much smaller than the northward component. A pair of regions with opposite but approximately equal perturbation are located mainly over the polar region; the eastward perturbation is located on the dawn side, 
dB Northward

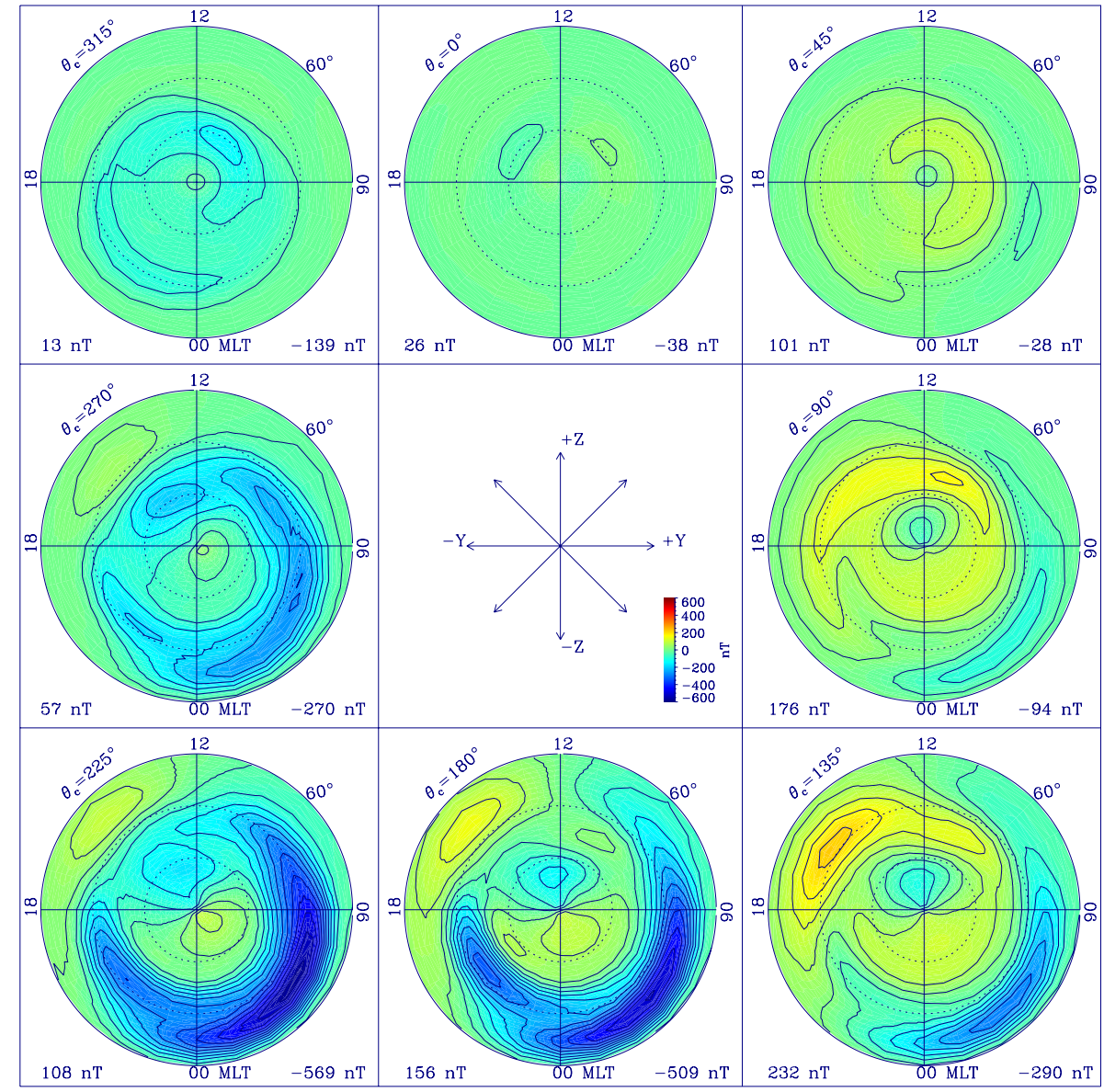

Fig. 3. Evolution of northward component of the geomagnetic perturbations as the IMF clock angle rotates. Peak values in the contour map are indicated in the bottom corner in each polar plot, northward is specified as positive; The intensity of perturbation is characterized by the color interpreted in the color bar; Latitude circles are plotted at $10^{\circ}$ intervals, the outer boundary of each plot is at $60^{\circ}$ latitude.

the westward perturbation is located on the dusk side, and the perturbation increases as the IMF clock angle rotates to southward. The location of the peak values are near $80^{\circ}$ magnetic latitude. These are roughly consistent with the statistical results. The evolution of the vertical component (Fig. 5) of the geomagnetic perturbations in simulation also generally agrees with the statistical results. When IMF is due northward, the two cells are located above $80^{\circ}$ magnetic latitude with the downward perturbation locates on the dusk side, the upward perturbation locates on the dawn side; at $\theta_{\mathrm{c}}=45^{\circ}$ a round region with upward perturbation is located at the polar region, at dawn side of it there is a crescent region with downward perturbation, this is reversed for $\theta_{c}=315^{\circ}$; when IMF rotates southward, a pair of downward and upward perturbation regions on the dawn side correspond to the dawn side westward electrojet, and another pair of downward and upward perturbation regions on the dusk side correspond to dusk side eastward electrojet. All of these indicate that the evolution of the ECS as the rotation of the IMF clock angle derived from the MHD model are reasonable.
We extract the peak values of the perturbations at these eight IMF clock angles from both the modeled and the statistical results and show how they vary as the IMF clock angle changes in Fig. 6. From top to bottom, the graphs are plotted for (a) northward, (b) eastward and (c) vertical components. For the most dominate northward component (Fig. 6a), the simulation result follows the trend of the statistical result well, the simulated southward peak value as a function of $\theta_{\mathrm{c}}$ agrees with the observation with a high crosscorrelation coefficient of 0.969 ; the cross-correlation coefficient for the northward peak value is 0.855 . For the eastward component (Fig. 6b), the simulated variation trend of the eastward peak value agree with the observation pretty well with the highest cross-correlation coefficient of 0.976 among the coefficients for the peak values in six directions, while the coefficient of 0.618 for the westward peak value is the smallest among the six cross-correlation coefficients. The low cross-correlation coefficient for the westward peak vale is mainly caused when the simulation fails to predict the reversal at due northward IMF $\left(0^{\circ} / 360^{\circ}\right.$ clock angle). For 


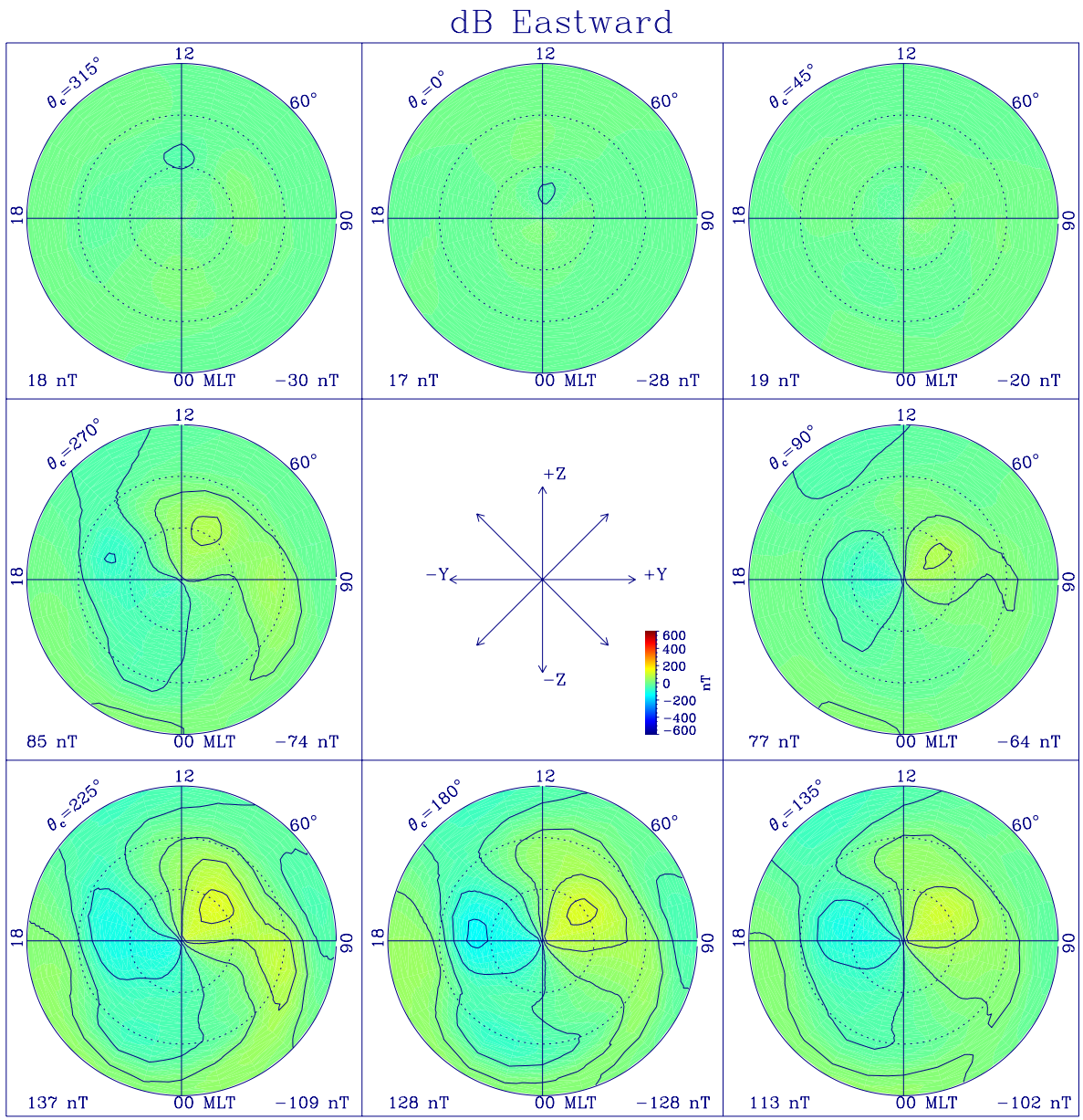

Fig. 4. Evolution of eastward component of the geomagnetic perturbations as the IMF clock angle rotates in the same format as Fig. 3 . Eastward is specified as positive.

the vertical component (Fig. 6c), the simulation captures the general trends of the downward and upward peak value as a function of the IMF clock angle with cross-correlation coefficients of 0.764 and 0.855 , respectively. The graphs imply that the simulation generally reproduces the variation tendency of the average ECS as a function of the IMF clock angle and the simulated current intensities are comparable to the observation.

Discrepancies between the simulation and the statistical results are not unexpected, since the MHD model does not take into account every physical process in the magnetosphere-ionosphere coupling system. A more detailed comparison between Fig. 3 and the observation show that the northward perturbation on the dusk sector plot in Weimer et al.(2010) (their Fig. 9) when $\theta_{\mathrm{c}}=270^{\circ}$ is roughly equal to the magnitude of the southward perturbation, while the northward perturbation on the dusk sector produced from the MHD model is too weak. The comparison of the patterns along with Fig. 6a indicates that the modeled northward perturbation on the dusk sector is smaller than the observation especially when the IMF is southward. This implies that the simulation fails to reproduce sufficient eastward equivalent current at the dusk sector. This may be caused by the lack of ring current in the MHD model, which is associated with the development of the eastward current, since it is generally believed that the eastward current is close to the partial ring current in the magnetosphere through region 2 FAC. Another disagreement is that the model fails to reproduce the reversals at due northward IMF orientation $\left(0^{\circ} / 360^{\circ}\right.$ clock angle) in the statistical results especially for the eastward component. It implies that the intensity of the ECS under clock angle $\theta_{\mathrm{c}}=0^{\circ}$ is underestimated in the simulation. The perturbations are located in the polar cap region when the clock angle is $0^{\circ}$, and the discrepancy may be caused by the ionospheric conductance model we used for this region. As introduced in Sect. 2.1, the Hall and Pedersen conductance over the dark polar cap region are given to be constants $2.0 \mathrm{~S}$ and $1.0 \mathrm{~S}$, respectively (Wang et al., 2011). Because of the conductance model adopted in the simulation, the increase of conductance in the region of the upward part of NBZ current system 


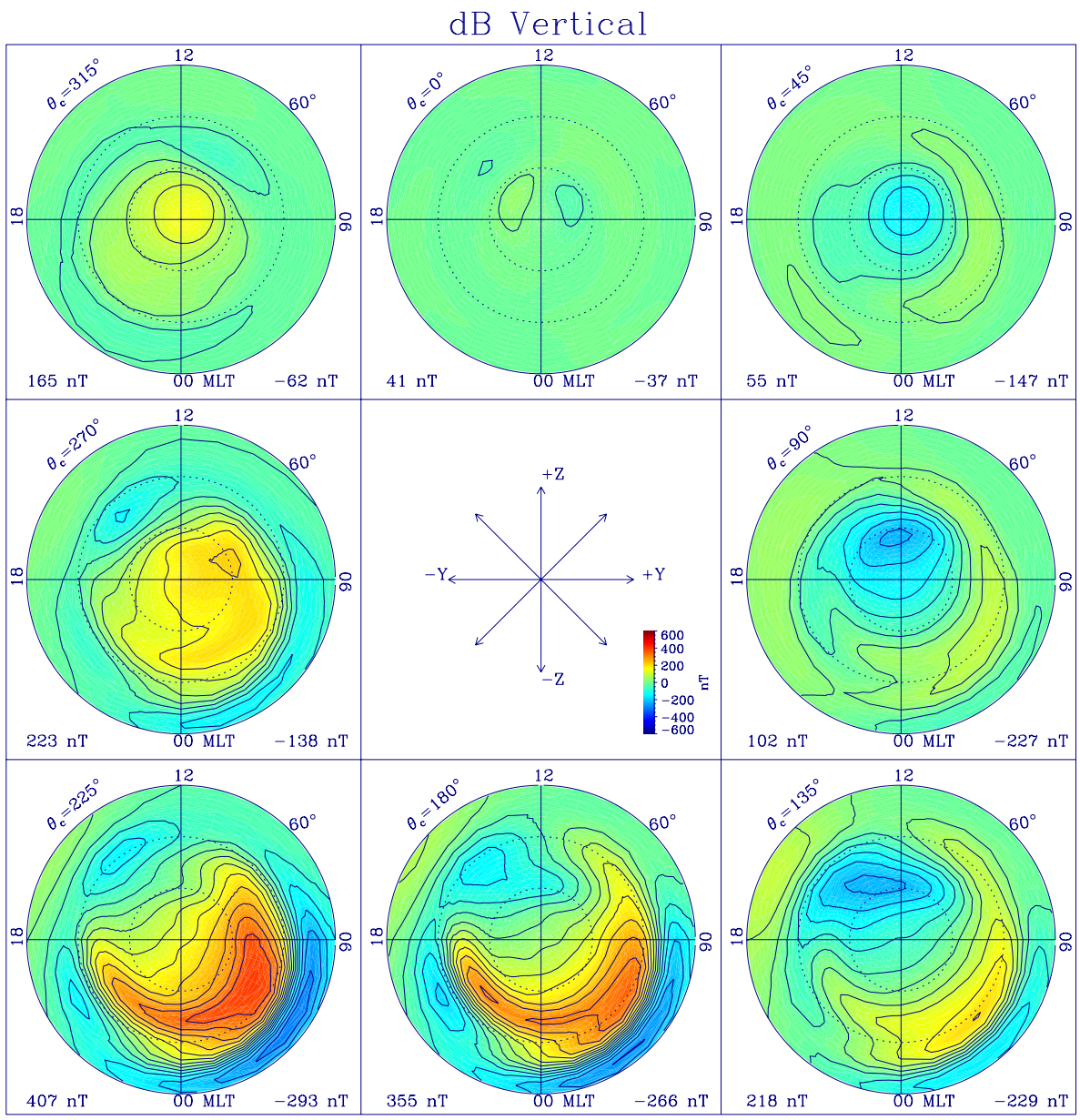

Fig. 5. Evolution of vertical component of the geomagnetic perturbations as the IMF clock angle rotates in the same format as Fig. 3 . Downward is specified as positive.

(Iijima et al., 1984) caused by electron precipitation is neglected in the simulation model when IMF is due northward, which means that the conductance in the model is lower than real conductance. The lower ionospheric conductance could cause lower ionospheric currents and NBZ currents over the polar cap region. It further causes the underestimation of the magnetic perturbations. The use of a more accurate conductance model over the polar cap region can be considered to improve the simulation results in the future.

\subsection{ECS as a function of southward IMF and solar wind velocity}

\subsubsection{Simulation results}

A series of patterns of average ECS under different solar wind conditions are also generated to examine the influence of the southward IMF magnitude and solar wind speed. We find that the general patterns are similar under the same IMF clock angle, while the intensity of the current is influenced by the IMF magnitude and solar wind velocity under south- ward IMF conditions. We extract the peak values of the eastward and westward currents and study how the current intensity change as the conditions vary. Figure 7 a shows the peak values of the currents as a function of the southward IMF magnitude $-B_{\mathrm{Z}}$, here the clock angle is fixed to $180^{\circ}$ (-z-orientation), $-B_{\mathrm{z}}$ increases from $0 \mathrm{nT}$ to $15 \mathrm{nT}$ with $5 \mathrm{nT}$ increments, $V_{\mathrm{x}}$ is fixed at $450 \mathrm{~km} \mathrm{~s}^{-1}, N$ is $7.5 \mathrm{~cm}^{-3}, T$ is set to $10^{5} \mathrm{~K}$, other parameters are 0 . Figure $7 \mathrm{~b}$ gives us the variation tendency of the peak values of currents as the solar wind velocity increases when IMF is fixed at $B_{\mathrm{Z}}=-5 \mathrm{nT}$, the velocity $V_{\mathrm{x}}$ increases from $350 \mathrm{~km} \mathrm{~s}^{-1}$ to $650 \mathrm{~km} \mathrm{~s}^{-1}$ with $100 \mathrm{~km} \mathrm{~s}^{-1}$ increments, other parameters keep constant with the above cases. Under southward IMF, as shown in Fig. 7, the intensities of both westward and eastward electrojet increase linearly with the increase of the magnitude of southward IMF and solar wind velocity, and the increase of the westward electrojet is much steeper than the eastward electrojet. In Fig. 7a, the westward peak value increases from about $0.1 \mathrm{~A} \mathrm{~m}^{-1}$ to $1.5 \mathrm{~A} \mathrm{~m}^{-1}$ as the southward IMF magnitude increases from $0 \mathrm{nT}$ to $15 \mathrm{nT}$, while the eastward 
(a) Northward

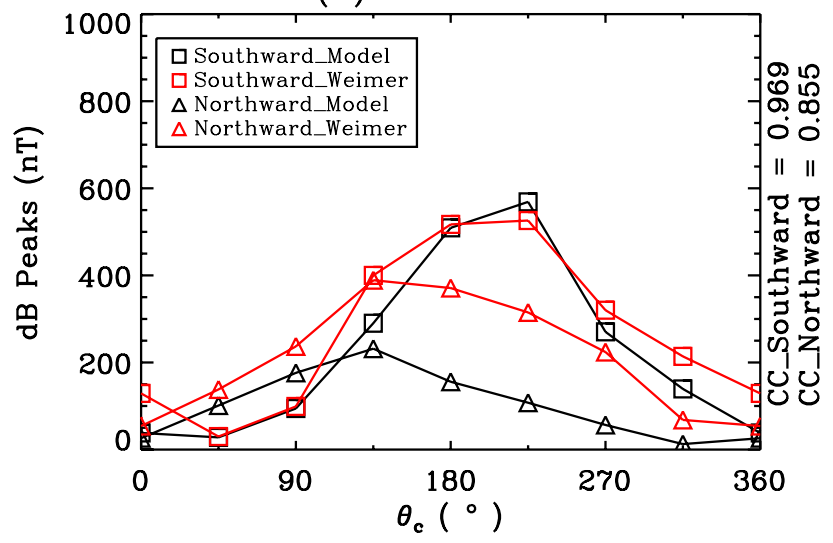

(b) Eastward

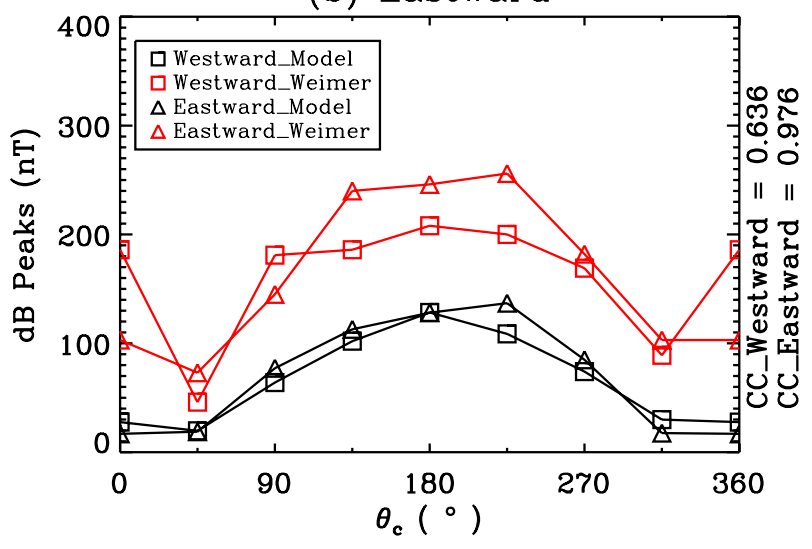

(c) Vertical

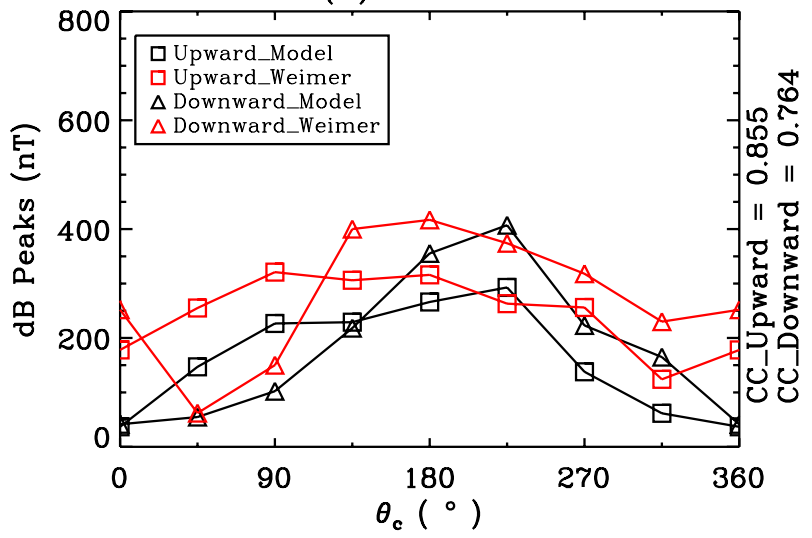

Fig. 6. Peak values of the geomagnetic perturbations as a function of the IMF clock angle $\theta_{\mathrm{c}}$. From top to bottom three graphs show the (a) northward, (b) eastward, (c) vertical components. Triangle and square symbols represent respectively the most positive and negative perturbation. Red symbols denote the statistical peak values from Weimer et al. (2010) and the black symbols denote the modeled peak values. Cross-correlation coefficients between the simulation and the observation are labeled on the right axis for each graph. peak value only increases from about $0.1 \mathrm{~A} \mathrm{~m}^{-1}$ to less than $0.5 \mathrm{~A} \mathrm{~m}^{-1}$; in Fig. $7 \mathrm{~b}$, the westward peak value increases from $0.4 \mathrm{~A} \mathrm{~m}^{-1}$ to $1.1 \mathrm{~A} \mathrm{~m}^{-1}$, while the eastward value increases from $0.2 \mathrm{~A} \mathrm{~m}^{-1}$ to $0.3 \mathrm{~A} \mathrm{~m}^{-1}$ and the solar wind speed $V_{\mathrm{X}}$ rises from $350 \mathrm{~km} \mathrm{~s}^{-1}$ to $650 \mathrm{~km} \mathrm{~s}^{-1}$.

\subsubsection{Comparison with the statistical results}

We then calculate the three components of geomagnetic perturbations from the simulated average ECS, and extract the peak values of perturbations to compare them with the statistical results. Figure 8 shows the change of the peak values of the geomagnetic perturbations with the increase of the southward IMF magnitude, the statistical values are extracted from Fig. 12 of Weimer et al. (2010). Except for the southward IMF, other IMF and solar wind parameters in the simulation and the statistical work are generally consistent. The IMF clock angle is $180^{\circ}$ and $V_{\mathrm{x}}$ is $450 \mathrm{~km} \mathrm{~s}^{-1}$ in both works, $N$ is set to $7.5 \mathrm{~cm}^{-3}$ in the simulation, the dipole tilt angle corresponds to equinox in both work. The simulation results indicate that for the most dominate northward component, the southward perturbation increases more steeply than the northward perturbation, which correspond to the steeply increased westward electrojet; the westward and eastward perturbations increase slowly with similar magnitude; the vertical component increases linearly with the rise of the magnitude of the southward IMF, while the downward peak values are consistently larger than the upward peaks, which implies that the clockwise equivalent current cell which is located at the dawn side is more intense than the counterclockwise current cell located at the dusk side.

Since the dipole tilt angle in simulation is the same as the observation, quantitative comparison can be conducted in this section. Based on the values in Fig. 8, except for the cross-correlation coefficient (CC), two other metrics - normalized root mean square difference (nRMS error) and prediction efficiency (PE) - are calculated to estimate the model performance. The definition of the nRMS and PE are

$\mathrm{nRMS}=\sqrt{\frac{\left\langle\left(\Delta B_{\mathrm{mod}}-\Delta B_{\mathrm{obs}}\right)^{2}\right\rangle}{\left\langle\Delta B_{\mathrm{obs}}^{2}\right\rangle}}$
$\mathrm{PE}=1-\frac{\left\langle\left(\Delta B_{\mathrm{mod}}-\Delta B_{\mathrm{obs}}\right)^{2}\right\rangle}{\sigma_{\text {obs }}^{2}}$,

where $\Delta B$ is the magnetic perturbation for the observation ( $\left.\Delta B_{\text {obs }}\right)$ or the modeled value $\left(\Delta B_{\text {mod }}\right),\langle\ldots\rangle$ indicates arithmetic mean, and the $\sigma_{\mathrm{obs}}^{2}$ denotes the variance of the observed data. The perfect value for the nRMS error is zero, and less than 1 means the observed and calculated perturbation have the similar trend with some offset. The nRMS error greater than 1 implies the calculated perturbation may miss the basic trend of the actual perturbation, and there exists a large deviation between them. The PE equal to 1 indicates perfect prediction, while the $\mathrm{PE}$ equal to 0 means that the performance of the model is as good as that of a model that uses the 

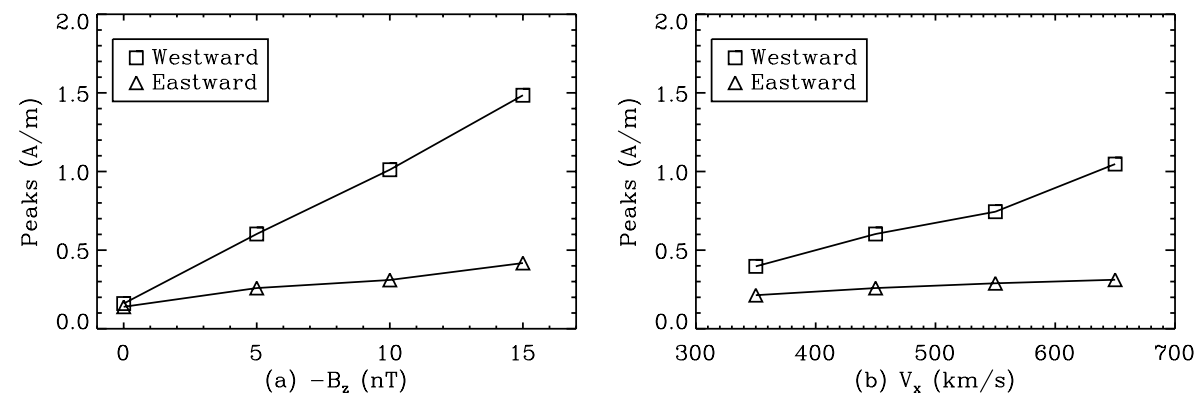

Fig. 7. (a) Peak values of the westward electrojet and eastward electrojet as a function of the southward IMF magnitude. (b) Peak values of the westward electrojet and eastward electrojet as a function of the solar wind velocity. In the graphes the peak values of eastward electrojet are indicated by triangle symbols, the peak values of westward electrojet are indicated by square symbols.

Table 1. Three metrics computed on the basis of the values in Fig. 8.

\begin{tabular}{lrrrrrr}
\hline & North & South & East & West & Down & Up \\
\hline CC & 0.951 & 0.980 & 0.894 & 0.926 & 0.997 & 0.997 \\
nRMS & 0.341 & 0.117 & 0.259 & 0.368 & 0.108 & 0.258 \\
PE & -0.784 & 0.916 & -0.060 & -0.925 & 0.926 & 0.478 \\
\hline
\end{tabular}

mean value of the observation as a predictor. After interpolation of the simulation results, we calculate the three metrics (CC, nRMS and PE) on the basis of the values in Fig. 8 and list them in Table 1. All of the cross-correlation coefficients are very high, and the lowest value is 0.894 for the eastward peak value and the highest value is 0.997 which is almost 1 ; the lowest nRMS error is 0.108 for the downward peak value and the largest value is 0.368 for the westward peak value; the highest PE is 0.916 , which is very close to perfect value 1 , for the southward peak value. All of these indicate that simulation results match pretty well with the statistical results, the MHD model reproduces not only the variation tendency but also the intensities of the perturbations quite well.

Figure 9 shows the variations of peak values of the geomagnetic disturbances with the increase of the solar wind speed $V_{\mathrm{x}}$. Peak values of the statistical work are extracted from Fig. 13 of the paper of Weimer et al. (2010). The IMF and solar wind conditions are also generally consistent in the two works. In the same format as Fig. 8, Fig. 9 indicates that the simulation results are also in good agreement with the statistical results. Table 2 presents the three metrics calculated on the basis of the values in Fig. 9. The cross-correlation coefficients for the peak values in six directions are all larger than 0.8 , nRMS errors are relative low, and the best PE is 0.691 for the southward peak value.

\section{Discussion and summary}

In this study we investigated the effect of solar wind conditions such as the IMF clock angle, southward IMF magnitude and solar wind velocity on the average ECS by using
Table 2. Three metrics computed on the basis of the values in Fig. 9.

\begin{tabular}{lrrrrrr}
\hline & North & South & East & West & Down & Up \\
\hline CC & 0.980 & 0.964 & 0.835 & 0.883 & 0.957 & 0.894 \\
nRMS & 0.284 & 0.134 & 0.308 & 0.492 & 0.181 & 0.271 \\
PE & -3.635 & 0.691 & -1.499 & -2.502 & 0.584 & -2.540 \\
\hline
\end{tabular}

the PPMLR-MHD model (Hu et al., 2007). We presented the change of average ECS pattern as the rotation of the IMF clock angle, we found that the ECS is weak when the IMF is northward, and the two-cell current system dominates when the IMF turns southward; when the IMF is southward, the intensity of the westward current is larger than the eastward current. We also show that, within the range of the solar wind parameters we investigated, the intensities of the westward and eastward current increases linearly as the rise of the southward IMF magnitude and solar wind speed under southward IMF, and the increase of the westward current is much sharp than the eastward current. Due to the lack of the direct observation-based ECS at certain interplanetary conditions, we compared the three components of geomagnetic perturbations on the surface of earth represented by the simulated average ECS with the statistical geomagnetic perturbations (Weimer et al., 2010) under similar IMF and solar wind conditions. Comparison results indicate that the simulated patterns for the geomagnetic perturbations evolve as the rotation of the IMF clock angle in the same way as the observationbased statistical results, and the peak values of the perturbations as a function of the IMF clock angle, southward IMF magnitude and solar wind velocity derived from the global MHD model match with the statistical results quite well. All of these imply that the average ECS as a function of the IMF and solar wind derived from the PPMLR-MHD model are reasonable. In addition, the solar wind plasma density $N$ could affect the intensity of the electrojets. Shue and Kamide (2001) showed a positive relationship between the plasma density and the intensity of the auroral electrojets by studying a period of time during which there are several density 


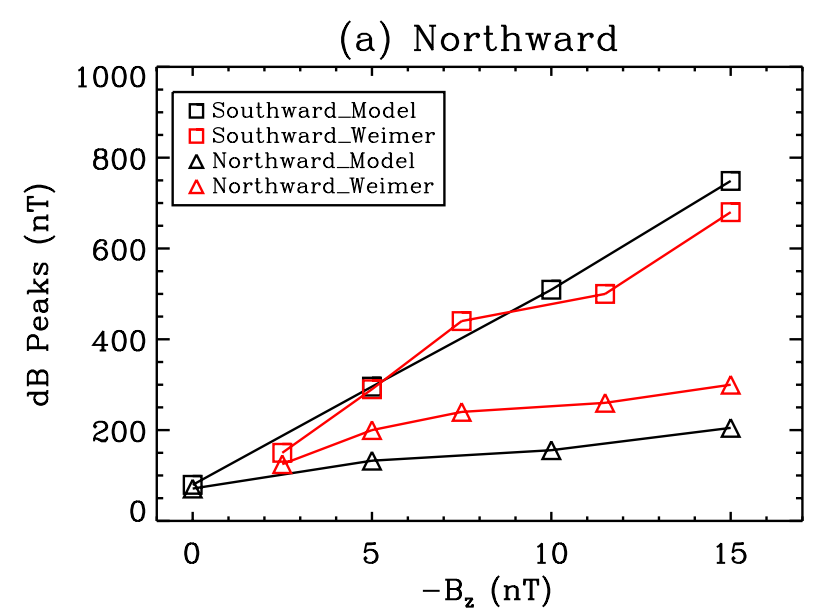

(b) Eastward

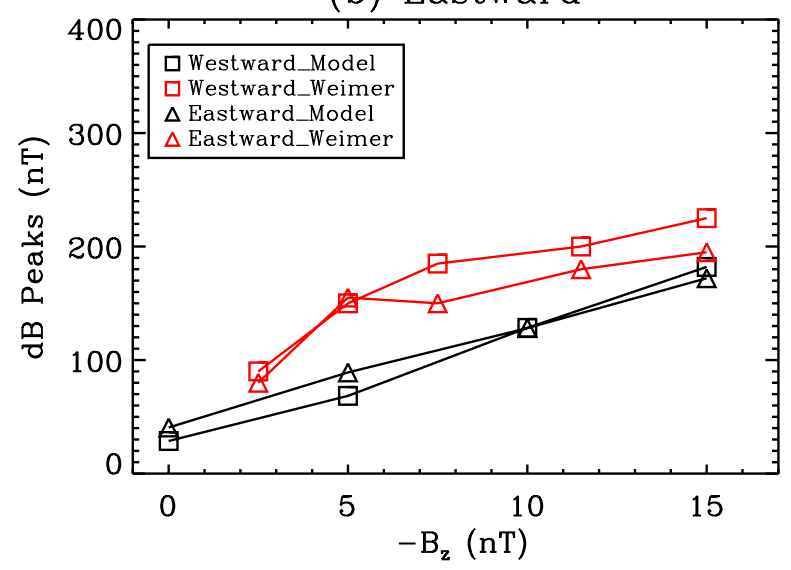

(c) Vertical

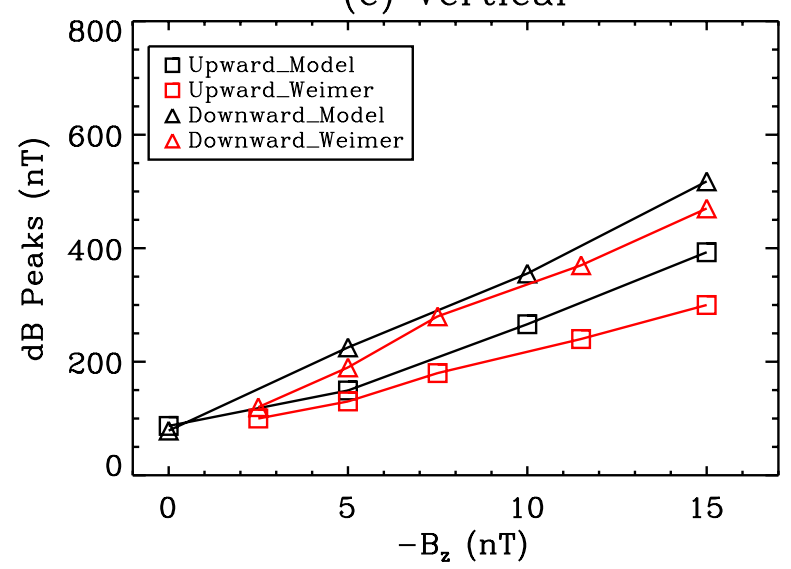

Fig. 8. Peak values of the geomagnetic perturbations as a function of the the southward IMF magnitude. From top to bottom three graphs show the (a) northward, (b) eastward, (c) vertical components. Triangle and square symbols represent the most positive and negative perturbation, respectively. Black lines denote the simulation results, red lines denote the statistical results. (a) Northward

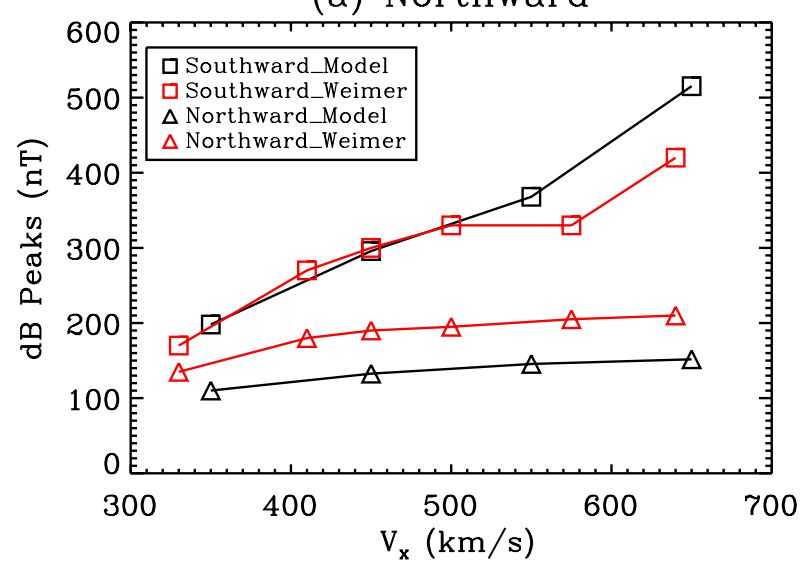

(b) Eastward

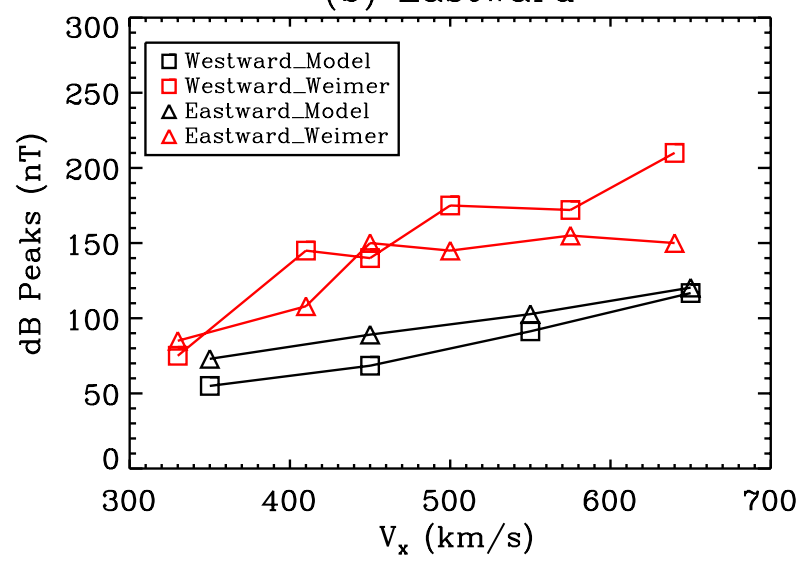

(c) Vertical

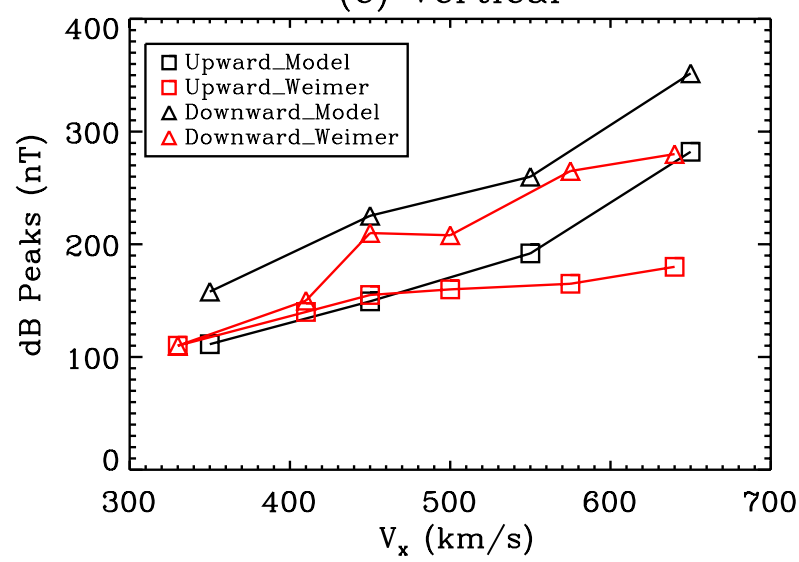

Fig. 9. Peak values of the geomagnetic perturbations as a function of the solar wind velocity. The three graphs show the (a) northward, (b) eastward, (c) vertical components. Triangle and square symbols represent the absolute values of most positive and negative perturbation, respectively. Black lines denote the simulation results, red lines denote the statistical results. 
enhancements while other solar wind parameters were relatively constant. They also pointed out that when the IMF is directed southward, the density effect is dominant in the westward electrojet. We have run the model to investigate the effect of the plasma density on the average ECS, the results also support the conclusions of Shue and Kamide (2001) qualitatively. We do not present the results in the paper due to the lack of the observation-based counterpart.

As expected, there were some discrepancies between the simulation and the statistical work, which give us some clues about how to improve the model. The model failed to reproduce sufficient eastward electrojet on the dusk sector, which requires that the model must include the effects of the ring currents. When the IMF is due northward, the simulation underestimated the intensity of the ECS, which is mainly located at the polar cap region. We considered that this discrepancy should attribute to the conductivity model we applied in the polar cap region. Application of more accurate conductance model in this region is expected to improve the model performance. The dipole tilt angle always stays at zero in the simulation in this study, so the results show the average ECS under certain IMF and solar wind condition without considering the seasonal effect. This effect could also be taken into account.

The average ECS as a function of the solar wind conditions derived from the global PPMLR-MHD model gave us a clear view of the coupling between the solar wind and the ionospheric current. With respect to space weather, this work may have economic implications due to the potential impact on the long conductor technical systems on the ground such as power grids and oil or gas pipelines. Like many ionospheric electrodynamic models in which the ionospheric field aligned current or electric potential are parameterized by the IMF and solar wind conditions, the average pattern of ECS derived in this study cannot be applied during short-period highly dynamic phenomenon substorms (e.g., Papitashvili et al., 2002; Weimer, 1995). It is noted that the underestimation of the eastward electrojet on the dusk sector is the main limitation to predict the ECS for the present model. However, the average ECS prediction from the MHD model is useful to show when and where the GIC events are most likely to take place under the interplanetary condition, since the large $d B / d t$, which are associated closely with the GIC, always occur within the regions of westward ionospheric electrojets (Viljanen and Tanskanen, 2011), this is similar to the terrestrial weather forecasts, which cannot give a prediction of the timing and locations of each individual lightning strike, the forecasters could give relative good forecast about the region where thunderstorms are likely to occur (Weimer, 2005a).

Acknowledgements. We wish to thank Y. Q. Hu and J. Kan for their helpful discussions. This work was supported by 973 program 2012CB825602, NNSFC grants 41231067, 40921063, and in part by the Specialized Research Fund for State Key Laboratories of China.
Topical Editor K. Hosokawa thanks V. P. Papitashvili and one anonymous referee for their help in evaluating this paper.

\section{References}

Ahn, B. H., Richmond, A. D., and Kamide, Y.: An ionospheric conductance model based on ground magnetic disturbance data, J. Geophys. Res., 103, 14769-14780, doi:10.1029/97JA03088, 1998.

Boteler, D. H. and Pirjola, R. J.: The complex-image method for calculating the magnetic and electric fields produced at the surface of the Earth by the auroral electrojet, Geophys. J. Int., 132, 31-40, 1998.

Chapman, S. and Bartels, J.: Geomagnetism, vol. 2, Oxford Univ. Press, London, 1940.

Friis-Christensen, E., Kamide, Y., Richmond, A. D., and Matsushita, S.: Interplanetary magnetic field control of high-latitude electric fields and currents determined from Greenland magnetometer data, J. Geophys. Res., 90, 1325-1338, 1985.

Fukushima, N.: Generalized theorem for no ground magnetic effect of vertical currents connected with pedersen currents in the uniform-conductivity ionosphere, Rep. Ionos. Space Res. Jpn., 30, 35-40, 1976.

Hu, Y. Q., Guo, X. C., and Wang, C.: On the ionospheric and reconnection potentials of the earth: Results from global MHD simulations, J. Geophys. Res., 112, A07215, doi:10.1029/2006JA012145, 2007.

Iijima, T., Potemra, T. A., Zanetti, L. J., and Bythrow, P. F.: LargeScale Birkeland Currents in the Dayside Polar Region During Strong Northward IMF: A New Birkeland Current System, J. Geophys. Res., 89, 7441-7452, 1984.

Janhunen, P.: GUMICS-3 a global ionosphere-magnetosphere coupling simulation with high ionospheric resolution, in: Environment Modeling for Space-Based Applications, vol. 392, pp. 233239, 1996.

Kamide, Y., Kanamitsu, M., and Akasofu, S. I.: A new method of mapping worldwide potential contours for ground magnetic perturbations- Equivalent ionospheric current representation, J. Geophys. Res., 81, 3810-3820, 1976.

Kamide, Y., Richimond, A. D., and Matsushita, S.: Estitmation of ionospheric electric fields, ionospheric currents, and fieldaligned currents from ground magnetic records., J. Geophys. Res., 86, 801-813, doi:10.1029/JA086iA02p00801, 1981.

Lyon, J. G., Lopez, R. E., Goodrich, C. C., Wiltberger, M., and Papadopoulos, K.: Simulation of the March 9, 1995, substorm: Auroral brightening and the onset of lobe reconnection, Geophys. Res. Lett., 25, 3039-3042, doi:10.1029/98GL00662, 1998.

Moen, J. and Brekke, A.: The solar Flux influence on quiet time conductances in the auroral ionosohere, Geophys. Res. Lett., 20, 971-974, doi:10.1029/92GL02109, 1993.

Papitashvili, V. O., Christiansen, F., and Neubert, T.: A new model of field-aligned currents derived from high-precision satellite magnetic field data, Geophys. Res. Lett., 29, 1683, doi:10.1029/2001GL014207, 2002.

Pettigrew, E. D., Shepherd, S. G., and Ruohoniemi, J. M.: Climatological patterns of high-latitude convection in the Northern and Southern hemispheres: Dipole tilt dependencies and interhemispheric comparisons, J. Geophys. Res., 115, A07305, doi:10.1029/2009JA014956, 2010. 
Pirjola, R. and Viljanen, A.: Complex image method for calculating electric and magnetic fields produced by an auroral electrojet of finite length, Ann. Geophys., 16, 1434-1444, doi:10.1007/s00585-998-1434-6, 1998.

Powell, K. G., Roe, P. L., Linde, T. J., Gombosi, T. I., and De Zeeuw, D. L.: A solution-adaptive upwind scheme for ideal magnetohydrodynamics, J. Comput. Phys., 154, 284, doi:10.1006/jcph.1999.6299, 1999.

Pulkkinen, A., Amm, O., and Viljanen, A.: Ionospheric equivalent current distributions determined with the method of spherical elementary current systems, J. Geophys. Res., 108, 1053, doi:10.1029/2001JA005085, 2003.

Raeder, J., McPherron, R. L., Frank, L. A., Kokubun, S., Lu, G., Mukai, T., Paterson, W. R., Sigwarth, J. B., Singer, H. J., and Slavin, J. A.: Global simulation of the geospace environment modeling substorm challenge event, J. Geophys. Res., 106, 381395, doi:10.1029/2000JA000605, 2001a.

Raeder, J., Wang, Y. L., Fuller-Rowell, T. J., and Singer, H. J.: Global simulation of space weather effects of the Bastille Day storm, Solar Phys., 204, 325-338, 2001 b.

Ridley, A., Crowley, G., and Freitas, C.: An empirical model of the ionosphere potential, Geophys. Res. Lett., 27, 3675-3678, 2000.

Shue, J. H. and Kamide, Y.: Effects of solar wind density on auroral electrojets, Geophys. Res. Lett., 28, 2181-2184, doi:10.1029/2000GL012858, 2001.

Untiedt, J. and Baumjohann, W.: Studies of polar current systems using the IMS Scandinavian magnetometer array, Space Sci. Rev., 63, 245-390, 1993.
Viljanen, A. and Tanskanen, E.: Climatology of rapid geomagnetic variations at high latitudes over two solar cycles, Ann. Geophys., 29, 1783-1792, doi:10.5194/angeo-29-1783-2011, 2011.

Wang, C., Zhang, J. J., Tang, B. B., and Fu, S. Y.: Comparison of equivalent current systems for the substorm event of 8 March 2008 derived from the global PPMLR-MHD model and the KRM algorithm, J. Geophys. Res., 116, A07207, doi:10.1029/2011JA016497, 2011.

Weimer, D. R.: Models of high-latitude electric potentials derived with a least error fit of spherical harmonic coefficents, J. Geophys. Res., 100, 19595-19607, doi:10.1029/95JA01755, 1995.

Weimer, D. R.: Predicting surface geomagnetic variations using ionospheric electrodynamic models, J. Geophys. Res., 110, A12307, doi:10.1029/2005JA011270, 2005a.

Weimer, D. R.: Improved ionospheric electrodynamic models and application to calculating Joule heating rates, J. Geophys. Res., 110, A05306, doi:10.1029/2004JA010884, 2005b.

Weimer, D. R., Clauer, C. R., Engebretson, M. J., Hansen, T. L., Gleisner, H., Mann, I., and Yumoto, K.: Statistical maps of geomagnetic perturbations as a function of the interplanetary magnetic field, J. Geophys. Res., 115, A10320, doi:10.1029/2010JA015540, 2010.

Zhang, J. J., Wang, C., and Tang, B. B.: Modeling geomagnetically induced electric field and currents by combining a global MHD model with a local one-dimensional method, Space Weather, 10, S05005, doi:10.1029/2012SW000772, 2012. 